\title{
Drying and Wetting in Finite Element Shallow-Water Flows
}

\author{
Onno Bokhove \& Djoko Wirosoetisno \\ O. Bokhove, Department of Applied Mathematics, University of Twente, Enschede, The Netherlands \\ o.bokhove@math.utwente.nl \\ D. Wirosoetisno, Bloomington, U.S.A.
}

\section{INTRODUCTION}

We consider space discontinuous Galerkin finite element discretizations of the symmetric or onedimensional shallow-water equations. These hyperbolic equations arise as shallow layer approximations in (geophysical) fluid dynamics, and are used in the prediction of river flooding and near-shore surf-zone dynamics in particular. In many of these practical applications, part of the boundary of the domain is defined by a free boundary where the water depth is zero, while other parts of the boundary are impenetrable walls, for example. Due to the movement of this free boundary or water line over topography, drying and wetting occurs. The validation and application of a novel numerical finite element method for drying and wetting by shallow-water dynamics is the subject of the present paper.

Discontinuous Galerkin finite element schemes (see, e.g., Yan \& Shu, 2002, and references therein) have several advantages: i) The structure of the scheme allows inclusion of a variable order of accuracy in each element (so-called $p$-adaptivity). ii) It is straightforward to refine or coarsen the elements (socalled $h$-adaptivity). iii) The scheme is local as the data communication occurs entirely through the faces between the elements or at boundaries. Especially this property is used to deal efficiently with free boundaries in the shallow-water equations. But it also allows for efficient parallel implementation. Moreover the implementation of in- and outflow boundary conditions is efficient and accurate due to the local nature of the scheme. Disadvantages are that the scheme is slightly more complex, and that more degrees of freedom are involved relative to finite-volume or finitedifference schemes.

We have validated the algorithm and code by comparing the numerical solutions with various onedimensional exact solutions in which wetting and drying occurs, and multiple wet patches emerge: Riemann problems with drying (e.g., Toro, 1999), the Carrier-Greenspan (1958) solution, the parabolic bowl solution (Young, 1986), and the PeregrineWilliams (2001) solution. Furthermore, we show a complex simulation with wave breaking, run-up and topping over a dike to display the potential of the numerical discretization.

In the following sections, we present the shallow water model ( $\$ 2)$, the space discontinuous Galerkin finite element approximation ( $(3)$, the drying and wetting algorithm ( $\S 4)$, the additional numerical dissipation $(\S 5)$, and the numerical results $(\S 6)$. We finalize with a conclusion and discussion in $\S 7$.

\section{SHALLOW WATER EQUATIONS}

The symmetric (or one-dimensional) shallow-water equations consist of the following mass and momentum equations

$$
\begin{aligned}
\partial_{t} h+\partial_{x}(h u) & =0 \\
\partial_{t}(h u)+\partial_{x}\left(h u^{2}+g h^{2} / 2\right) & =-g h \partial_{x} h_{b}+S_{2}{ }^{\prime} \\
\partial_{t}(h v)+\partial_{x}(h u v) & =S_{3}
\end{aligned}
$$

with the (depth-averaged) horizontal velocity field $(u, v)=(u(x, t), v(x, t))$, the depth $h(x, t)$ of the layer as function of the horizontal coordinate $x$ and time $t$. The additional (topographic and dissipation) terms $S_{2}=-g h \partial_{x} h_{b}+S_{2}{ }^{\prime}$ and $S_{3}$ can in general depend on $x$ and $t$ explicitly, and implicitly on the variables $u, v, h$. The topography at $z=h_{b}(x)$ is measured from a horizontal reference level $z=0$. In addition, partial derivatives are denoted by $\partial_{t}=\partial / \partial t$ and $\partial_{x}=\partial / \partial_{x}$.

For the numerical formulation, we rewrite (1) concisely as

$$
\partial_{t} U+\partial_{x} F=S
$$


with state vector $U=(h, h u, h v)^{T}$, flux vector $F(U)=\left(h u, h u^{2}+g h^{2} / 2, h u v\right)^{T}$, and "source" vector $S=\left(0, S_{2}, S_{3}\right)^{T}$.

The flow domain $\Omega_{f}$ is a subset of a bounded domain $\Omega$ (here $[a, b]$ with $b>a$ ). It may be time dependent and has a boundary $\partial \Omega_{f}$. The boundary consists in general of a combination of fixed and free boundary segments (here nodes). At the fixed boundary segments, the boundary conditions specify the in- and outflow or no normal-flow conditions. The free boundary is specified by $h(x, t)=0$. The system (1) or (2) is completed with initial conditions $h_{0}=h(x, 0), u_{0}=u(x, 0), v_{0}=v(x, 0)$.

\section{FINITE ELEMENT APPROXIMATION}

The domain $\Omega_{f}$ is partitioned into elements $K_{k}$ with $k=1, \ldots, N$. In on dimension, element $K_{k}$ has two nodes or faces at $x=x_{k}(t)$ and $x=x_{k+1}(t)$. $\left|K_{k}\right|=$ $x_{k+1}-x_{k}$ with $x_{k+1}>x_{k}$ is the length of an element. Each element with $x \in\left(x_{k}, x_{k+1}\right)$ is mapped to a reference element $\hat{K}$ with coordinate $\zeta \in(-1,1)$. $\zeta= \pm 1$ at the right and left face, respectively. In principle, elements can be time dependent by allowing the nodes to move in time.

The variables $U$ and test functions $v$ are approximated by $U_{h}$ and $v_{h}$, respectively, using first-order polynomial approximations in each element $K_{k}$, as follows

$$
\begin{aligned}
U_{h}(x, t) & =\sum_{k}\left(\bar{U}_{k} \psi_{0, k}+\hat{U}_{k} \psi_{1, k}\right) \text { and } \\
v_{h}(x) & =\sum_{k}\left(\bar{W}_{k} \psi_{0, k}+\hat{W}_{k} \psi_{1, k}\right)
\end{aligned}
$$

with basis functions $\psi_{0, k}=1, \psi_{1, k}=\zeta$ defined in each reference element $\hat{K}_{k}$, in which $\bar{U}_{k}$ and $\hat{U}_{k}$ are the mean and the slope of the variable $U_{h}$ in each element. Characteristic of the discontinuous Galerkin finite element approximation is that the approximation $U_{h}(x, t)$ is discontinuous across element faces, since the basis functions $\psi_{i, k}(x, t)$ jump to zero at the edges of each element. Thus, in $K_{k}$ we have $U_{h}=\bar{U}_{k}+\zeta \hat{U}_{k}$.

In general, test function $v_{h}$ is also discontinuous across elements. When we multiply (2) by the test function $v_{h}=v_{h}(x, t)$ and integrate by parts, a weak formulation is obtained

$$
\begin{aligned}
\sum_{k=1}^{N}\{\quad & \int_{K_{k}} v_{h} \partial_{t} U_{h} \mathrm{~d} x+ \\
& {\left[F\left(x_{k+1}\right) v_{h}\left(x_{k, R}^{-}\right)-F\left(x_{k}\right) v_{h}\left(x_{k, L}^{+}\right)\right]-} \\
& \left.\int_{K_{k}^{-}} F \partial_{x} v_{h} \mathrm{~d} x-\int_{K_{k}} S v_{h} \mathrm{~d} x\right\}=0,
\end{aligned}
$$

where $v_{h}\left(x_{k, R}^{-}\right)=\lim _{x \uparrow x_{k, R}} v_{h}(x, t), \quad v_{h}\left(x_{k, L}^{+}\right)=$ $\lim _{x \downarrow x_{k, L}} v_{h}(x, t)$, and $x_{k, R}=x_{k+1}$ and $x_{k, L}=x_{k}$. In the integration by parts, the derivative $\partial_{x} v_{h}$ yields three contributions: two delta functions arising from discontinuities in $v_{h}$ at the element faces, and the (constant) derivative in the interior of elements. In the integral $\int_{K_{k}^{-}} F \partial_{x} v_{h} \mathrm{~d} x$ the element edges are excluded, as denoted by $K_{k}^{-}$, since we have already picked up the flux contributions from the jumps in $v_{h}$ at the element edges. Since $U_{h}$ is discontinuous, the fluxes $F\left(x_{k+1}\right)$ at the faces are not well-defined and will depend on the values $F\left(\bar{U}_{k}+\hat{U}_{k}\right)$ for $\zeta=$ 1 in element $K_{k}$ and $F\left(\bar{U}_{k+1}-\hat{U}_{k+1}\right)$ for $\zeta=-1$ in element $K_{k+1}$, that is, in the elements immediately left and right of the face $x_{k+1}$. We will replace the fluxes $F\left(x_{k+1}\right)$ at the faces by a numerical flux $\tilde{F}\left(\bar{U}_{k}+\hat{U}_{k}, \bar{U}_{k+1}-\hat{U}_{k+1}\right)$.

Since $v_{h}$ is arbitrary, we take $v_{h}=\bar{W}_{k}$ and $v_{h}=\hat{W}_{k}$. After substitution into the weak formulation (5) and evaluation of several of the (elemental) integrals, we obtain the discretization

$$
\begin{aligned}
& \frac{d}{d t}\left(\left|K_{k}\right| \bar{U}_{k}\right)=-\left(\tilde{F}_{v}\left(\bar{U}_{k}+\hat{U}_{k}, \bar{U}_{k+1}-\hat{U}_{k+1}\right)-\right. \\
& \left.\tilde{F}_{v}\left(\bar{U}_{k-1}+\hat{U}_{k-1}, \bar{U}_{k}-\hat{U}_{k}\right)\right)+ \\
& \frac{\left|K_{k}\right|}{2} \int_{-1}^{1} S\left(U_{h}, \zeta, t\right) \mathrm{d} \zeta \\
& \frac{d \hat{U}_{k}}{d t}=-\frac{3}{\left|K_{k}\right|}\left(\tilde{F}\left(\bar{U}_{k}+\hat{U}_{k}, \bar{U}_{k+1}-\hat{U}_{k+1}\right)+\right. \\
& \left.\tilde{F}\left(\bar{U}_{k-1}+\hat{U}_{k-1}, \bar{U}_{k}-\hat{U}_{k}\right)\right)+ \\
& \hat{U}_{k}\left(V_{k, R}-V_{k, L}\right) /\left|K_{k}\right|+ \\
& \frac{3}{\left|K_{k}\right|} \int_{-1}^{1} F \mathrm{~d} \zeta+\frac{3}{2} \int_{-1}^{1} S \zeta \mathrm{d} \zeta \\
& \left|K_{k}\right|=x_{k, R}-x_{k, L} \\
& \frac{d x_{k,(R, L)}}{d t}=V_{k,(R, L)},
\end{aligned}
$$

where $\tilde{F}_{v}$ is a numerical discretization of $F(U)-V U$ as, in general, nodes $x_{k,(R, L)}$ can move in time with a certain speed $V_{k,(R, L)}$.

The numerical flux at the faces is the HLLC flux of Toro et al. (1994), determined by considering an approximate Riemann problem based on the values immediately left and right of the faces. We use the 
total variation diminishing third-order Runge-Kutta scheme of Shu \& Osher (1989) as time discretization.

\section{DRYING AND WETTING}

The fluid in the domain $\Omega_{f}$ is divided into one or more distinct patches of fluid in a bounded region $\Omega$ separated by dry regions, see Fig. 1. In one dimension, each patch has a left and right boundary. When required, the boundary conditions at the external boundaries $x=a$ and $x=b$ can be various, such as periodic and open boundary conditions, the latter with prescribed in- and outflow conditions depending on the characteristics, and no-normal flow through moving or fixed walls.

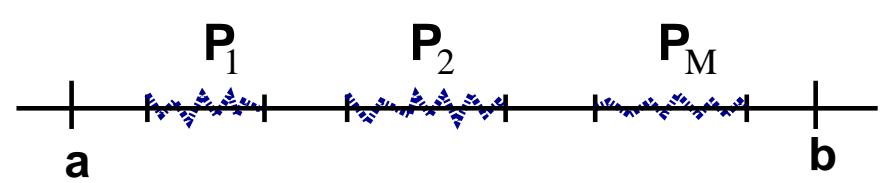

Figure 1: A sketch of the $M$ patches of $\Omega_{f}$ within the regular finite element space $\Omega$.

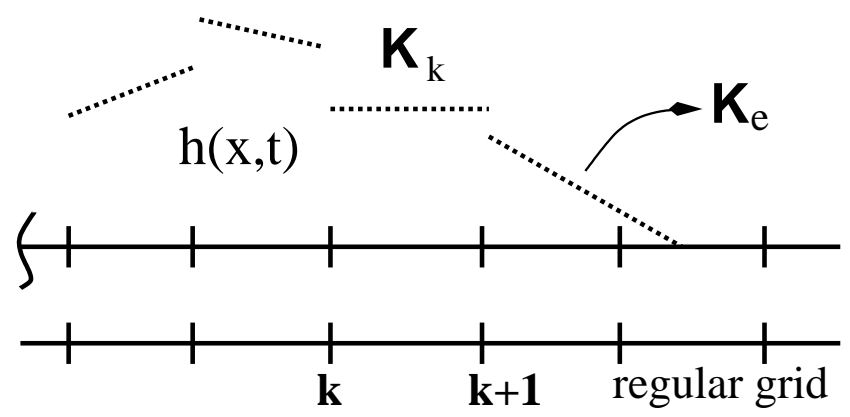

Figure 2: A patch in the finite element space with a right edge element $K_{e}$. Denoted is the height field $h(x, t)$ for the case with linear basis functions. Note that in $K_{e}$ on the right we have $h=0$.

In one dimension a dry-wet boundary is demarcated by a particle on the left or right of the patch where the depth $h=0$. There are three situations to distinguish: i) a patch of fluid moves along with a drywet boundary on the left, on the right, or at both sides; ii) a patch of fluid breaks up into two patches in which case we may have to define two new particles; and iii) two patches of fluid merge into one patch of fluid.

For elements in the interior of patches, we fix the nodes so that $V_{k,(L, R)}=0$, while at the left and right edge of a patch where $h=0$, see Fig. 2, the edge node can move so that $V_{k,(L, R)}=u_{L, R}$, respectively, is the local fluid speed. The discretization (6) includes the cases for both the fixed interior and moving edge elements. Since $h=0$ at the edges, the slope $\hat{U}_{k}= \pm \bar{U}_{k}$ at the left and right elements, respectively. Hence, only the equations for the mean $\bar{U}_{k}$ remain relevant in the edge elements.

In order to maintain the mostly Eulerian nature of the numerical scheme, i) we split an edge element when it becomes too large (larger than say 1.6 times the size of an underlying regular element $\left|K_{k r}\right|$ ), and ii) we merge an edge element with its neighbor when the former becomes too small (smaller than say 0.4 times $\left|K_{k r}\right|$ ) after each completed time step [see Fig. 3]. Therefore, the number of elements in a patch with at least one free boundary may change over time.

a)

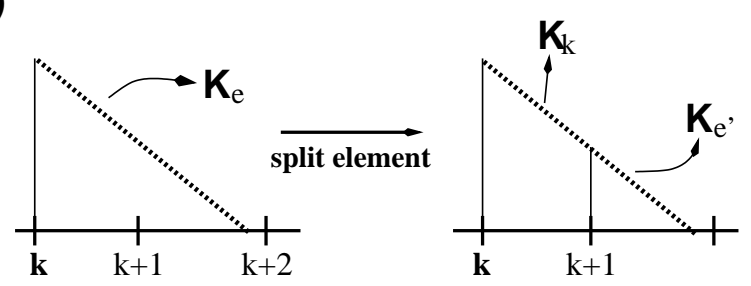

b)

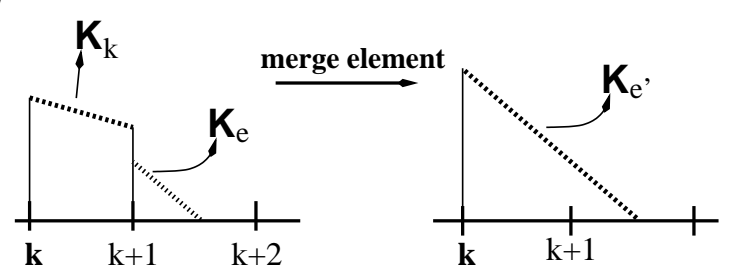

Figure 3: Element a) splitting and b) merging is sketched for the height field for the case with linear basis functions.

In addition to element merging and splitting discussed above, patches may also merge and split. When the depth of the fluid becomes zero in the interior of a patch or when a splitting criterion is met, the patch is split into two patches with free boundary conditions at the splitting point. In the absence of source terms $S$, the splitting criterion derives optimally from a Riemann splitting criterion for a the flat bottom case, that is, at an edge splitting occurs when $u_{l}+\sqrt{g h_{l}} \leq u_{r}-\sqrt{g h_{r}}$ or approximately, as $h \rightarrow 0$, when $u_{l} \leq u_{r}$ with $u_{l, r}$ and $h_{l, r}$ the values of $u$ and $h$ left and right of a face, respectively. Alternatively, the Riemann problem may be considered for a locally constant bottom slope. However, to avoid negative or zero water depths as much as possible we actually use an ad hoc splitting criterion, namely $\bar{U} \pm \hat{U}<\epsilon_{d} \Delta t \bar{U}$ for a left (+-sign) and right front (--sign), respectively. We have taken $\epsilon_{d}=0.001$, for example, or smaller. The idea is that when the water depth approaches zero at the front, the depth at the front becomes a small fraction of the mean water depth in that element.

The time step of this explicit scheme is restricted on the one hand by the CFL condition associated with the 


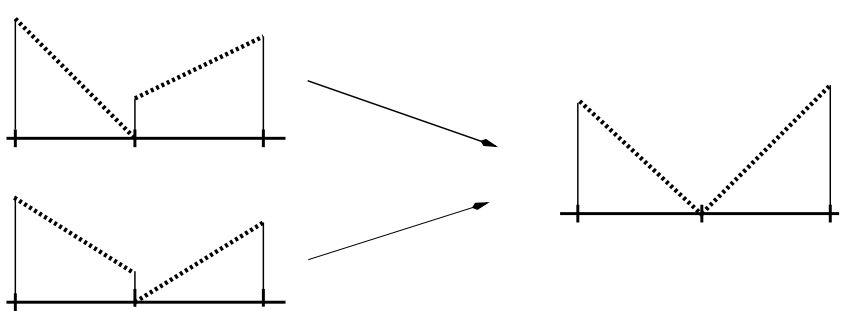

Figure 4: Patch splitting is sketched for the height field.

a)
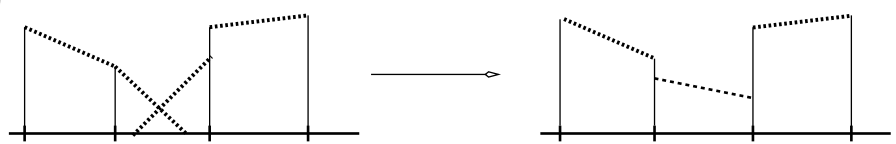

b)

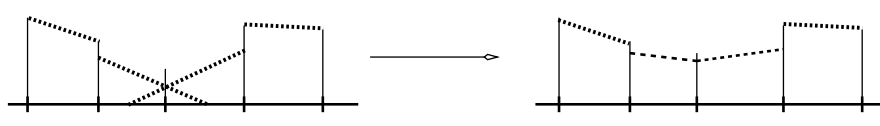

Figure 5: Patch merging is sketched for the height field for two generic cases where edges overlap a) in one element and b) in two elements.

eigenvalues $|u \pm \sqrt{g h}|$, and on the other hand by requiring that the mean values $\bar{H}_{k}$ of $h$ in each element remain positive. This time step restriction to ensure positivity of the mean depth essentially follows from a maximum principle analysis of the Runge-Kutta time discretization and the HLLC flux for the depth or continuity equation. Since the edge elements can not become very small due to the element splitting process, the time step restriction to ensure positive mean depth does not become too restrictive.

Finally, element and patch splitting and merging is handled after each time step. At intermediate time steps of the Runge-Kutta scheme, patches can therefore overlap, and when necessary we limit slopes $\hat{U}_{k}$ to $\pm \bar{U}_{k}$ to avoid negative depths. L'Hopitals rule is used to calculate $u=(h u) / h$ when $h \rightarrow 0$ at element faces while the mean depth is guaranteed to be positive $\bar{H}_{k}>0$ following our time step restriction.

\section{NUMERICAL DISSIPATION}

For smooth solutions of (1) in which the variables are single-valued functions of $x$ the discretization does not require additional numerical dissipation. However, we consider the shallow water equations extended to permit local discontinuities such as bores and hydraulic jumps in which mass and momentum is conserved, but energy is dissipated. This motivated the choice of the particular formulation (1) in terms of $h, h u$ and $h v$, which is the starting point to derive the well-known energy-dissipating jump or Rankine-
Hugoniot relations (see, e.g., Toro, 1999).

When discontinuities are present in solutions of (1), the numerical finite-element solutions without additional dissipation will exhibit steep gradients with spurious oscillations. Although, the numerical scheme remains stable and robust, we generally wish to limit these spurious oscillations near discontinuities. However, the addition of a numerical dissipation (following, e.g., Jaffre et al, 1995) or a slope limiting procedure (see, e.g., Burbeau et al, 2001) in the whole domain degrades the accuracy near the free dry-wet boundary. This degradation can be so severe, that the water keeps sticking erroneously against the bottom. Ideally, extra dissipation or slope limiting must be restricted to those (steep) numerical gradients that are the counterparts of discontinuities in the continuous case.

So far, we have used the discontinuity detector of Krivodonova et al (2003) in combination with a Froude number criterion. The discontinuity detector of Krivodonova et al (2003) is based on the jumps in the value of $h$ at the faces of each element where fluid enters the element, divided by the maximum norm of the depth in an element times $\left|K_{k}\right|$ for the first order polynomials used here. In deriving this detector, Krivodonova et al (2003) used the error estimate that the numerical jumps at faces with inflow are $O\left(\left|K_{k}\right|^{3}\right)$ for smooth flows and $O\left(\left|K_{k}\right|\right)$ when genuine discontinuities are present (for first-order polynomials). The Froude number criterium considers a jump in $h$ between elements to be a genuine discontinuity when the Froude number minus one $F_{r}-1=$ $|u| / \sqrt{g h}-1$ is of opposite sign across a face, since the flow changes from supercritical to subcritical at a jump. Experience sofar indicated that this amalgamated shock indicator in combination with the slope limiter of Burbeau et al (2001) is least destructive: discontinuities are smoothed to some extent without destroying the accuracy at free boundaries too severely. So far, the dissipation by Jaffre et al (1995) in combination with this combined shock indicator was less successful.

To unify all the numerical results, we use the slope limiter only upon shock indication. However, for the simulation of smooth flows, more accuracy is reached when no extra dissipation is added.

Clearly, better dissipation schemes are desired to localize dissipation to effectively avoid spurious oscillations at genuine discontinuities. Alternatively, sufficient physical dissipation in the momentum equations may also eliminate spurious oscillations. In addition, the proposed drying and wetting algorithm can in principle be extended to dispersive shallow water (or Boussinesq) equations with a similar continuity equation. 


\section{VALIDATION AND RESULTS}

It turns out that each of the seven presented numerical solutions poses different requirements on the numerical scheme and the extra numerical dissipation. In all cases, we have scaled the equations, giving (1) with $g=1$ and $S_{2}{ }^{\prime}=S_{3}=0$. The last simulation shown is the only one without an exact solution and combines some of the features presented in the exact solutions.

First, we consider a case without drying, a moving bore reflecting against a wall at $x=1$. Preliminary simulations, for snapshots see Fig. 6, reveal that the numerical accuracy is of order one, $O(\Delta)$ with $\Delta$ the minimum element size, due to the precense of a discontinuity. Using slope limiting everywhere would yield smooth jump profiles everywhere. However, the unified more selective dissipation does yield oscillations. For a steady shock the applied unified dissipation does smoothen the jump effectively.
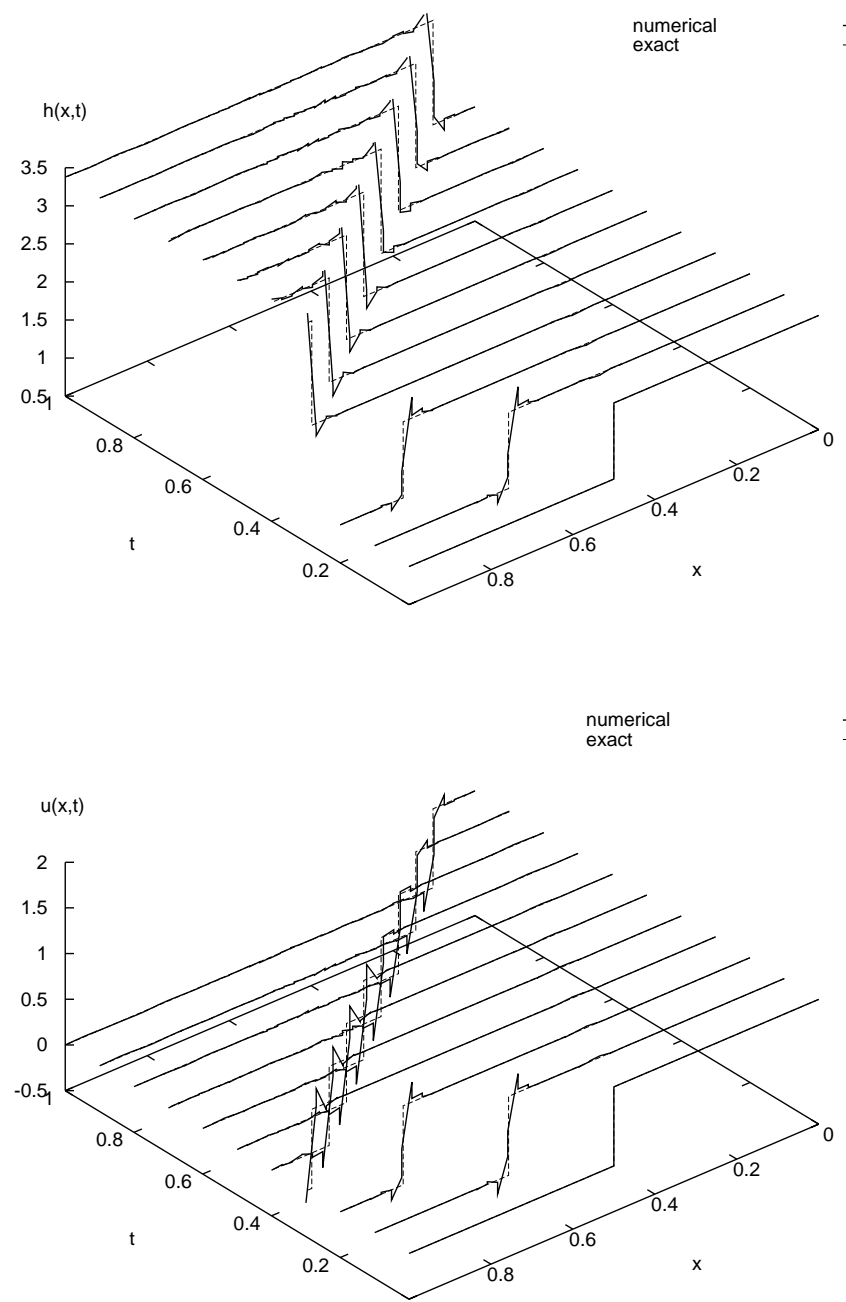

Figure 6: Free surface and velocity profiles are shown as function of space and time for the moving and reflected jump problem. Solid lines display the numerical solution, and dashed lines the exact solution. 20 elements are used and the unified slope limiter.

Second, for the dam break problem, preliminary simulations show that the order of accuracy is around $\Delta^{1.5}$ for the interior, but much lower $\Delta^{0.6}$ for the position of the front, see also Fig. 7. As a possible explanation, we note that the exact solution near the front in the dam break problem is quadratic in the velocity while in the edge element the velocity is only approximated by a constant.
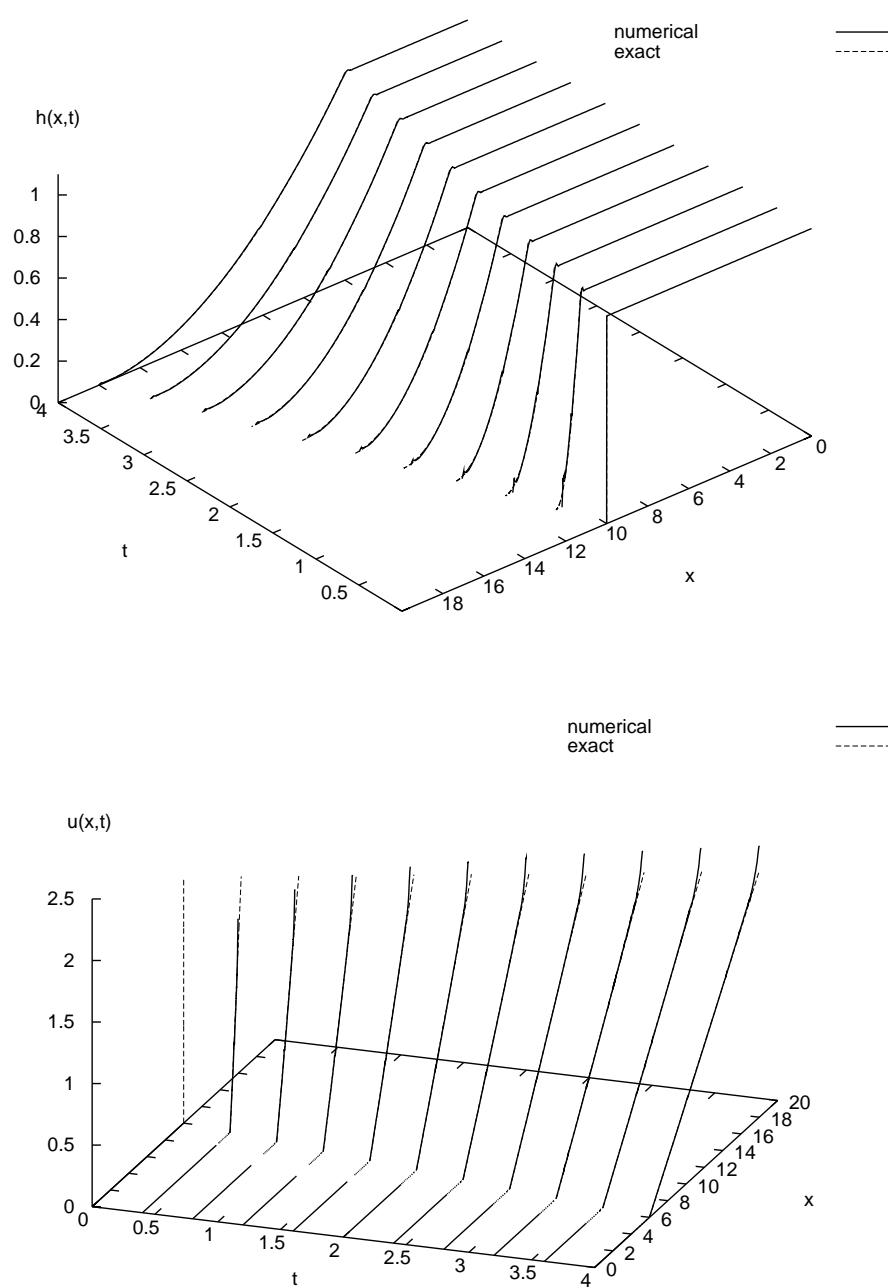

Figure 7: Free surface and velocity profiles are shown as function of space and time for the dam break problem. Solid lines display the numerical solution, and dashed lines the exact solution. 400 elements are used and the unified slope limiter.

Third, for certain divergent initial velocities, the Riemann problem results in a dry patch. Preliminary simulations, see also Fig. 8, show that the accuracy is of the order $\Delta^{1.5}$ overall and $\Delta^{0.6}$ at the front.

Fourth, preliminary numerical simulations without extra numerical in a parabolic bowl reveal that the numerical accuracy is of $O\left(\Delta^{2}\right)$ in comparison with exact solutions (Young, 1986) as expected for smooth solutions. The use of the unified slope limiter deteriorates the solution most particularly for this parabolic bowl solution, see Fig. 9, as the shock detection is too sensitive near the shorelines. The symmetry of the so- 

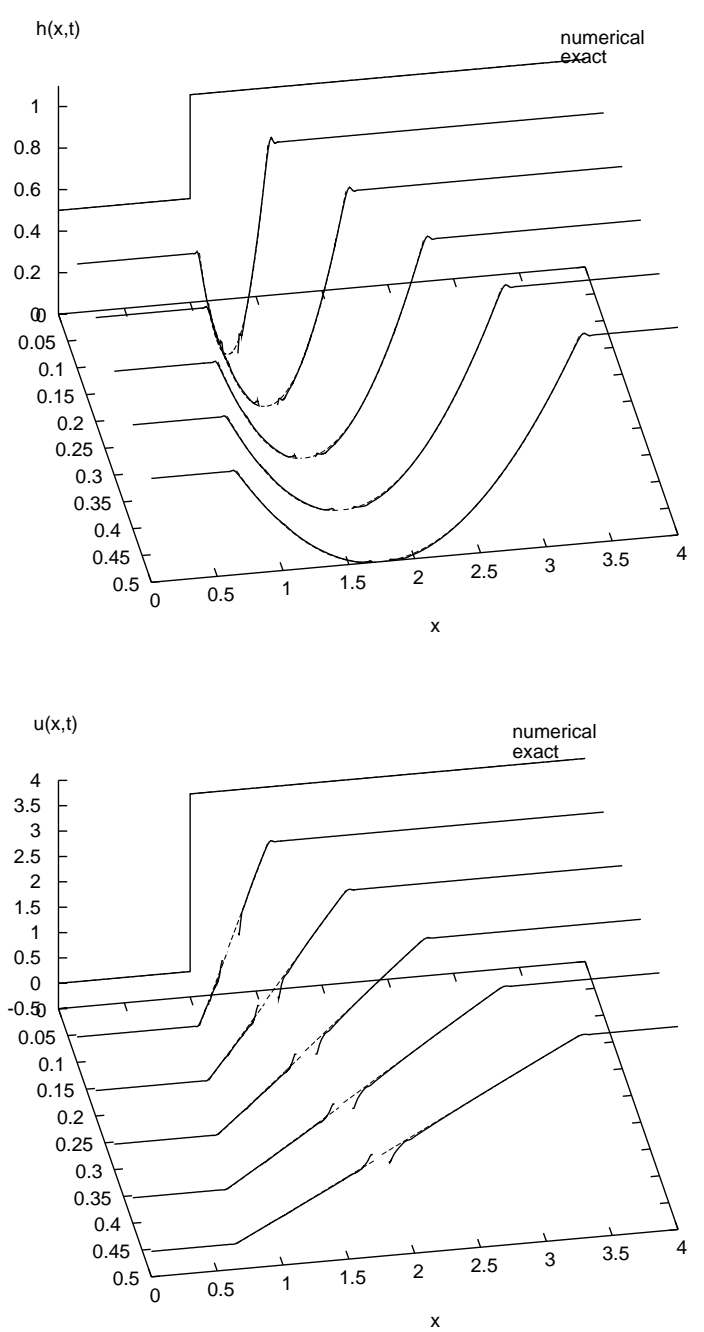

Figure 8: Free surface and velocity profiles are shown as function of space and time for the Riemann problem in which a dry patch emerges. Solid lines display the numerical solution, and dashed lines the exact solution. 400 elements are used and the unified slope limiter.

lution is useful in debugging the code.

Fifth, preliminary numerical simulations of the exact Carrier-Greenspan (1958) solution reveal that the numerical accuracy at the front is $\Delta^{0.9}$ and in the interior $\Delta^{2.8}$ without extra numerical dissipation. Plots of the height field $h(x, t)$, for example in Fig. 10 for the case with the unified slope limiter, show clearly that accuracy is lost where the gradient becomes very steep, which coincides with the region where the Jacobian of the hodograph transformation becomes small. From the plot of the velocity field $u(x, t)$, we clearly see that high velocities are not well approximated in the edge element. After all only the mean velocity is calculated by constraining $h$ and $h u$ to be zero at the front.

Sixth, preliminary simulations of the exact Peregrine-Williams solution of flow topping over a critical point, here the top of a dike, reveals that the accuracy in the interior before the patch breaks
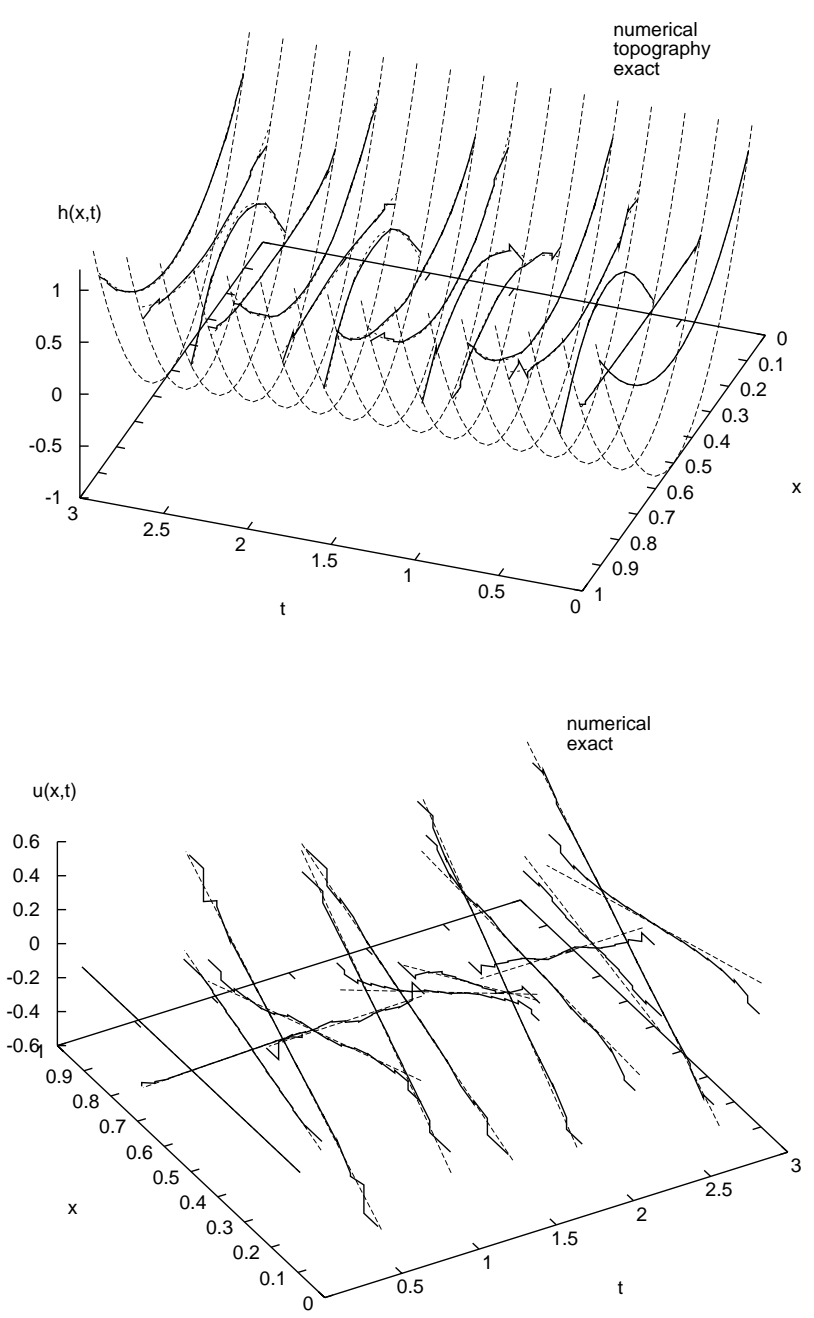

Figure 9: Free surface and velocity profiles are shown as function of space and time for the parabolic bowl problem. Solid lines display the numerical solution, (short) dashed lines the exact solution side of the dike, and long dashed lines the beach topography. 20 elements are used and the unified slope limiter.

is about $\Delta^{2}$ and drops to $\Delta^{1.5}$ after break up. The accuracy at the front rapidly degrades from $\Delta^{0.8}$ before break up to $\Delta^{0.4}$ after break up.

Finally, we show a complex simulation with wave steepening and breaking, run-up, topping over the critical top of a dike, and patch break up, in Fig. 12. Beyond the dike top the water rushes down in broken patches of fluid. A wave maker introduces sinusoidal waves offshore. Hence, we specify the state $U(x \uparrow 0, t)$, used in the flux HLLC calculation, by taking

$$
\begin{aligned}
& u(x \uparrow 0, t)=(k A / \omega) \sin (\omega t) \\
& h(x \uparrow 0, t)=H_{0}+A \sin (\omega t)
\end{aligned}
$$

with $H_{0}=1, k=\omega=3 \pi$ and $A=0.07$. Essentially depending on the characteristics, information flows in or out the domain. 

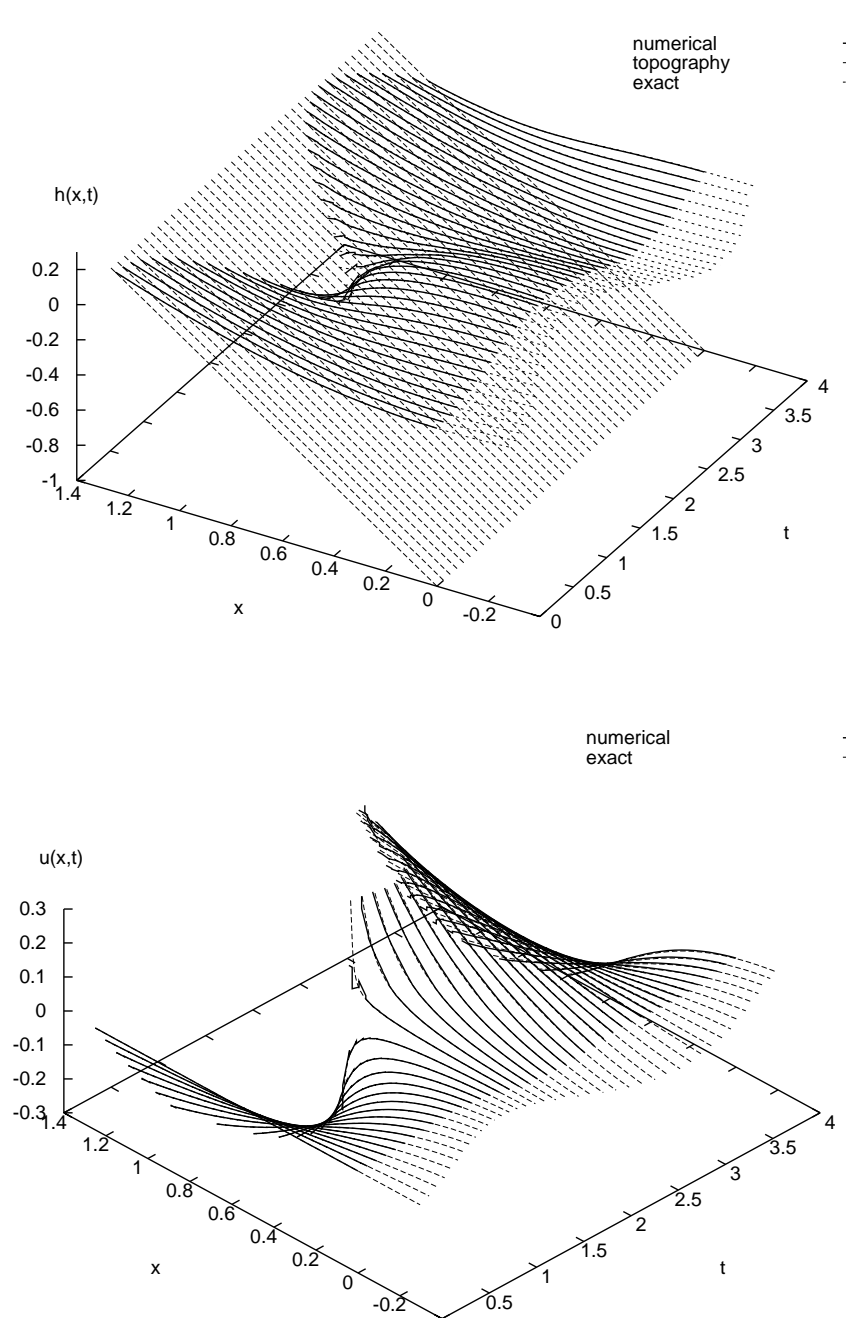

Figure 10: Free surface and velocity profiles for the Carrier-Greenspan test case for $N=40$ elements and the unified slope limiter. The beach topography is indicated by dotted lines.

In the simulation in Fig. 12, we see initially sinusoidal waves steepen to bores when they approach the shore. After a bore has formed the slope between bores is nearly plane parallel, and the dynamics behind the last bore thus resembles the initial condition of the idealized solution of Peregrine and Williams. In front of the last bore, the solution resembles the further evolution of the Peregrine-Williams solution. Due to the offshore driving of waves, multiple bores create multiple events of waves topping over the dike. On the downslope side of the dike, multiple patches of a thin layer of water rush down the slope with high speed before leaving the computational domain.

\section{CONCLUSION AND DISCUSSION}

A higher-order space discontinuous Galerkin finite element algorithm was developed for free boundary dynamics or drying and wetting in the symmetric shallow water equations. Positivity of the mean depth in each element was ensured under certain reasonable time step restrictions. The free boundary movement
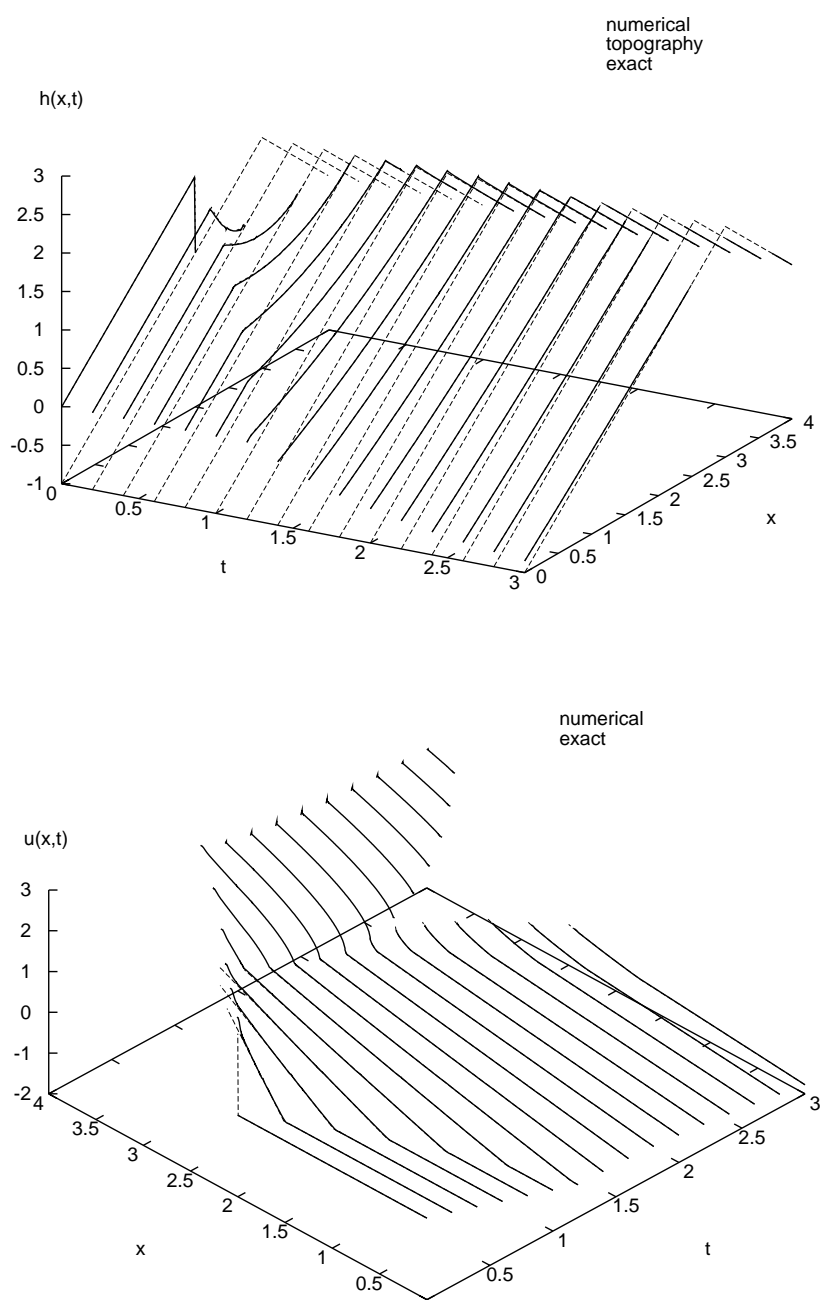

Figure 11: Free surface and velocity profiles are shown as function of space and time for the PeregrineWilliams solution. $N=320$ elements are used and the unified slope limiter.

and the appearance of dry patches in the middle of the fluid domain was handled by using the slope information of the depth. The robustness of the method was exemplified by a complex simulation of multiple shoaling and steepening water waves running up the seaward slope of a dike, overtopping, and then breaking up into two or more patches.

The numerical validations show that our method is second order in smooth cases and in the interior of the domain in the absence of discontinuities. It reduces to first order: in the presence of discontinuities such as shocks, hydraulic bores, or jumps; and at the free boundary. Moreover, it reduces to order half when (multiple) dry patches emerge. Inspection of the velocity profiles in the validation examples, shows that the velocity is only represented by its mean in the edge element at a free boundary, because we restrict the basis function to be linear near the front. The latter is realistic, except for certain initial conditions, but reduces the degree of freedom in the edge elements.

Local $p$-refinement at the edge element (such that 
the order of the basis and test function is raised) can increase the accuracy at the edge elements. Patch splitting reduces the accuracy. Using space time discontinuous Galerkin finite element methods may lead to more accurate patch splitting because the time of splitting can then be used explicitly.

Finally, work is underway to extend the onedimensional explicit space discontinuous Galerkin approximation to two dimensions, and also to implicit space time discontinuous Galerkin finite element approximations.

O.B. and D.W. gratefully acknowledge a fellowship from The Royal Dutch Academy of Arts and Sciences (KNAW) and a visitors' stipend from the Twente Institute of Mechanics of the University of Twente, respectively.

\section{REFERENCES}

[1] Burbeau, A., Sagaut, P., \& Bruneau, Ch.-H., A problem-independent limiter for higher-order Runge-Kutta discontinuous Galerkin methods. J. Comp. Phys. 169, 111-150, 2001.

[2] Carrier, G.F. \& Greenspan, H.P., Water waves of finite amplitude on a sloping beach. J. Fluid Mech. 4, 97-109, 1958.

[3] Jaffre, J, Johnson, C., \& Szepessy, A., Convergence of the discontinuous Galerkin finite element method for hyperbolic conservation laws. Math. Models and Methods in Appl. Sci. 5, 367-386, 1995.

[4] Krivodonova, L. Xin, J., Remacle, J.-F., Chevaugeon, N., \& Flaherty, J.E., Shock detection and limiting with discontinuous Galerkin methods for hyperbolic conservation laws. Manuscript, 21 pp., 2003.

[5] Peregrine, D.H. \& Williams, S.M., Swash overtopping a truncated plane beach. J. Fluid Mech. 440, 391-399, 2001.

[6] Shu, C-W. \& Osher, S., Efficient implementation of essentially non-oscillatory shock-capturing schemes II. J. Comp. Phys. 83, 32-78, 1989.

[7] Toro, E.F., Spruce, M., \& Speares, W., Restoration of the contact surface in the HLLRiemann solver. Shock Waves 4, 25-34, 1994.

[8] Young, W.R., Elliptical vortices in shallow water. J. Fluid Mech. 171, 101-119, 1986.

[9] Toro, E.F., Shock capturing methods for freesurface flows. Wiley, Toronto, 309 pp, 1999.

[10] Yan, J., \& Shu, C.-W., A local discontinuous Galerkin method for $\mathrm{KdV}$ type equations. Siam. J. Num. Analysis 54, 769-791, 2002.
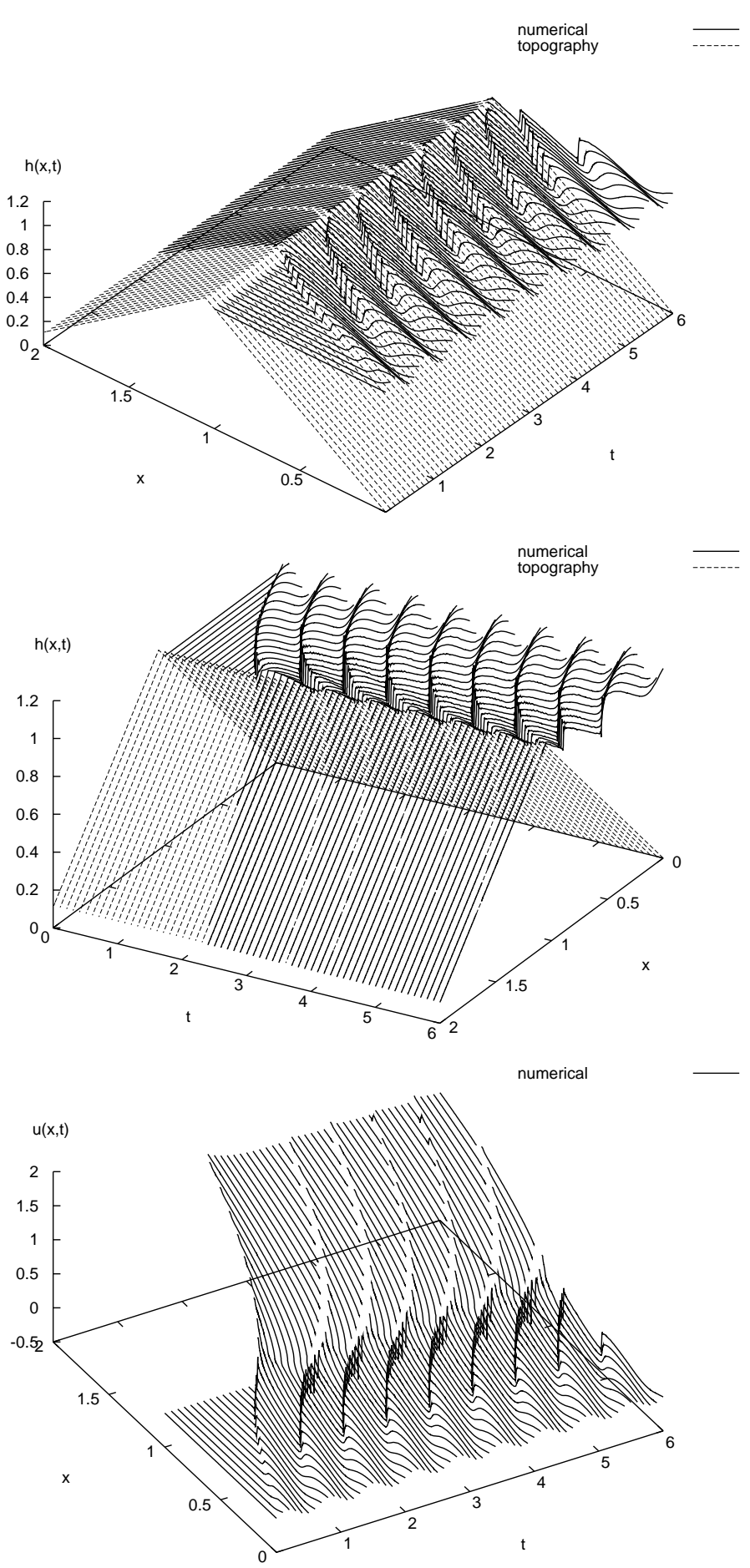

Figure 12: Two free surface profiles are shown from the front and back side of the dike, as well as velocity profiles for multiple waves steepening, runningup a dike and topping over a dike. 400 elements are used and the unified slope limiter. Note that the spacetime profiles eventually become periodic. One observes that the patches of water are rushing down the backslope very fast and are very thin. 